\title{
Mutational analysis of Smad7 in human cervical cancer
}

\author{
RAMKUMAR HARIHARAN ${ }^{1}$, JANKI MOHAN BABU ${ }^{1}$, REMA ${ }^{2}$ and M. RADHAKRISHNA PILLAI ${ }^{1}$ \\ ${ }^{1}$ Department of Molecular Medicine, Rajiv Gandhi Centre for Biotechnology; \\ ${ }^{2}$ Division of Surgical Oncology, Regional Cancer Centre, Thiruvananthapuram, Kerala, India
}

Received November 27, 2008; Accepted December 22, 2008

DOI: 10.3892/or_00000315

\begin{abstract}
Our aim was to carry out mutational analysis of Smad7 exon 4 that codes for the Smad7 MH2 domain in human cervical cancer tissues. This study is warranted since genetic abnormalities of components of the transforming growth factor- $\beta$ (TGF- $\beta$ )/Smad signaling system have been implicated in a variety of human cancers. Further, Smad7 is an important member of the Smad family that functions as a negative feedback regulator of TGF-ß responses. By direct sequencing, we screened 60 histopathologically confirmed human cervical carcinomas for mutations in exon 4 of Smad7 that encodes the $\mathrm{MH} 2$ domain of the protein, a region of the protein believed to be critical for receptor interaction. No mutations or aberrations could be identified in any of the 60 analyzed tumor samples. However, we identified a previously reported, heterozygous, silent $\mathrm{G}$ to $\mathrm{C}$ variant in codon 391 of Smad7 that was found twice in the set of 60 clinical samples. Thirty of the clinical samples analyzed in the study were positive for the presence of high-risk human papillomavirus (HPV) subtypes, while the rest were oncogenic HPV-negative. The two instances of the silent variant was found in oncogenic HPVnegative samples. We report mutational analysis of Smad7 in cervical cancer for the first time. Mutations and variants of Smad7 are unlikely to be of major significance to the pathogenesis of HPV-induced cervical cancer.
\end{abstract}

\section{Introduction}

Carcinoma of the uterine cervix remains the second most common form of female cancer worldwide (1). It has long been established that oncogenic types of the human papillomavirus (HPV) can act as etiological agents of cervical carcinoma (1-3). Presence of HPV type 16 and HPV type 18, the two major oncogenic HPV types, have been detected in the majority of cervical carcinomas and at least some of the molecular mechanisms underlying HPV-induced cervical

Correspondence to: Dr M. Radhakrishna Pillai, Department of Molecular Medicine, Rajiv Gandhi Centre for Biotechnology, Thycaud. P.O, Thiruvananthapuram, Kerala State 695 014, India E-mail: mrpillai@rgcb.res.in

Key words: Smad7, cervical cancer, human papillomavirus, mutation carcinogenesis are known $(4,5)$. Genetic abnormalities of the various components of the transforming growth factor- $\beta$ signaling pathway have been reported in a variety of cancers, including cervical cancer (6-10). The oncogenic nature of several key mutants of Smad2, Smad3 and Smad4 in cervical cancer has been reported (11-13).

Smads constitute a family of transcription factors that function as signal transducers in the transforming growth factor- $\beta$ (TGF- $\beta$ ) signaling system (14). The TGF- $\beta$ superfamily of secreted ligands includes the TGF- $\beta$ isoforms, bone morphogenetic proteins (BMPs), activins, nodal and growth/ differentiation factors. Ligand-induced activation of the type II TGF- $\$$ receptors containing a constitutively active kinase in its intracellular domain, results in trans-phosphorylation of the TGF- $\beta$ type I receptor. The phosphorylated type I receptor, in turn, phosphorylates their substrates, the Smads, for further propagating the signal. Activated Smads translocate to the nucleus, where they associate with various proteins and function as transcription regulators (15-17).

Smad1, 2, 3, 5 and 8 are receptor regulated, R-Smads, whereas Smad4 is the common or co-Smad that accompanies the R-Smads during their nuclear translocation. Smads have a conserved N-terminal MH1 domain that has DNA binding ability and a conserved C-terminal $\mathrm{MH} 1$ domain that mediates protein-protein interactions.

Smad6 and Smad7 are Inhibitory or I-Smads $(18,19)$. It has been shown that while Smad6 mainly functions to inhibit signals initiated by BMP ligands, nature of inhibition by Smad7 is more general (20-22).

In this study, we carried out mutational analysis of Smad7 exon 4 in 60 human cervical carcinoma tumors by direct sequencing. Exon 4 encodes the mad homology 2 (MH2) domain of Smad7 protein. The MH2 domain of Smad7 is known to provide specificity and affinity for Smad-TGF- $\beta$ type I R interaction (23). Screening for mutations/variants in exon 4 in clinical tumor samples is justified since it has been reported that specific point mutations, engineered in vitro in exon 4 (affecting MH2 domain) abolishes Smad-receptor interaction, thereby impairing $\operatorname{Smad} 7$ function (23).

However, we could not find any aberrations in the Smad7 exon 4 region in any of the analyzed set of tumor samples. But we observed a previously reported, heterozygous, silent $\mathrm{G}$ to $\mathrm{C}$ variant in codon 391 of $\mathrm{Smad} 7$ that was found twice in the set of 60 samples. Thirty of the cervical tumors analyzed in the study were positive for high-risk HPV subtypes while the other 30 tumors were oncogenic HPV-negative. Results from this study suggest that mutations in the Smad7 gene are 
Table I. Smad7 exon 4 (MH2 domain) mutations/variants in human cervical cancer.

\begin{tabular}{lccccc}
\hline Frequency of variant/mutation (40 samples tested) & Codon & Nucleotide & & Status & Amino acid change \\
\hline $2 / 60$ & 391 & CGG to CCG & Hetero/Hemi-zygous & Nil (silent) \\
\hline
\end{tabular}

The results of the mutation screening carried out in the set of 60 cervical cancer tumors.

A

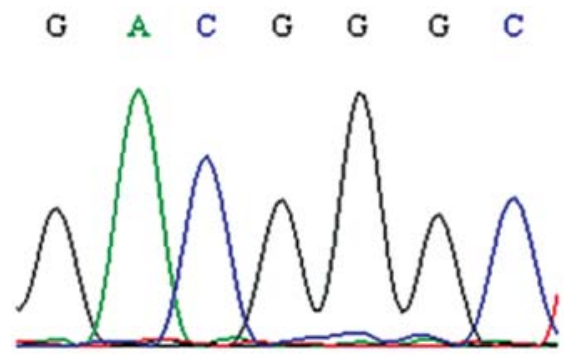

B

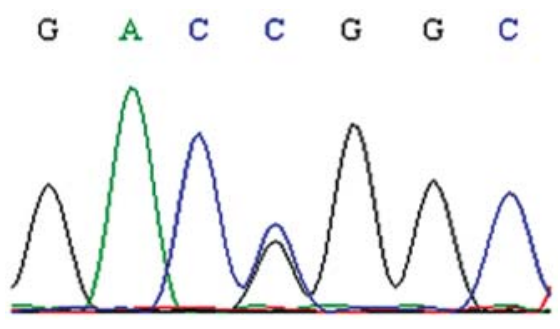

Figure 1. Chromatograms of the relevant regions of Smad7 discussed in the study. (A) The chromatogram of consensus Smad7 sequence with 'ACG' at codon 391. (B) The chromatogram of the heterozygous variant, with both 'C' and ' $\mathrm{G}$ ' occurring at third position of codon 391.

unlikely to be of major etiological significance in HPV-induced cervical cancer.

\section{Materials and methods}

The primary tumor tissues were from histopathologically confirmed cases of invasive squamous cell carcinoma of the cervix, obtained from patients attending Regional Cancer Centre, Thiruvananthapuram, India. The study was carried out after obtaining approval from the Institutional Review Board and Ethics Review Committee of Regional Cancer Centre, Thiruvananthapuram, India.

DNA extraction from tumor tissue. DNA was extracted from the tissue samples using the QIAamp DNA mini kit (Qiagen, Germany) as per the manufacturer's protocol.

DNA integrity check. The integrity of the purified genomic DNA was checked by amplifying with $\beta$-globin-specific primers. Primer sequences are as follows: Forward - 5'-GGT TGG CCA ATC TAC TCC CAG G-3' Reverse - 5'-GCT CAC TCA GTG TGGCAA AG-3'. The size of the amplicon was 540 bp. PCR amplification was carried out in $25 \mu 1$ volume containing 1X PCR buffer, 1 unit of Taq DNA polymerase (Promega), $1.5 \mathrm{mM} \mathrm{MgCl}_{2}, 200 \mu \mathrm{M}$ dNTPs (Promega), $10 \mathrm{pmol}$ each of forward and reverse primers and $1 \mu 1$ of the genomic DNA. PCR was done for 35 cycles with denaturation at $94^{\circ} \mathrm{C}$ for $30 \mathrm{sec}$; primer annealing at $60^{\circ} \mathrm{C}$ for $1 \mathrm{~min}$ and primer extension at $72^{\circ} \mathrm{C}$ for $1 \mathrm{~min} 30 \mathrm{sec}$. An initial denaturation of $94^{\circ} \mathrm{C}$ for 2 min was also included in the PCR reaction. The PCR products were analysed in $1.2 \%$ agarose gel.

HPV status and genotyping. PCR amplification using HPV consensus primers was carried out on the extracted DNA to determine the HPV status. Primer sequences are as follows: Forward primer - 5'-CGTCCMARRGGAAWACTGATC-3' and Reverse primer -5'-GCMCAGGGWCATAAYAATGG-3'. The size of the amplicon was $440 \mathrm{bp}$. PCR amplification was carried out in $25 \mu 1$ volume containing $1 \mathrm{X}$ PCR buffer, 1 unit of Taq DNA polymerase (Promega), $1.5 \mathrm{mM} \mathrm{MgCl}_{2}, 200 \mu \mathrm{M}$ dNTPs (Promega), 10 pmol each of forward and reverse primers and $1 \mu 1$ of the genomic DNA. The reaction was repeated for 35 cycles with denaturation at $94^{\circ} \mathrm{C}$ for $30 \mathrm{sec}$; primer annealing at $56^{\circ} \mathrm{C}$ for $1 \mathrm{~min}$ and primer extension at $72^{\circ} \mathrm{C}$ for $1 \mathrm{~min} 30 \mathrm{sec}$. An initial denaturation of $94^{\circ} \mathrm{C}$ for $2 \mathrm{~min}$ was also included in the PCR reaction. The PCR products were analysed in $1.2 \%$ agarose gel.

Type-specific primers were used to identify high-risk HPV genotypes in the clinical samples. Both the primer sequences as well as the PCR conditions for genotyping of the major high risk types of HPV (HPV 16, 18, 31, 33, 35, 52 and 58) that were used in this study have been reported earlier (24).

PCR amplification of Smad7 exon 4. The amplification was carried out with a pair of primers specific for exon 4 of Smad7. Primer sequences are as follows: Left primer 5'-GGC GGGTGTCTTCATTCTA-3' and right primer 5'-AGCAAG CACTCAGGAGGAAA-3'. The size of the amplicon was $696 \mathrm{bp}$. Sequencing of the amplified product tested the specificity for amplification. PCR amplification was carried out in $25 \mu \mathrm{l}$ volume containing 1X PCR buffer, 1 unit of Pfu-Taq DNA polymerase, $1.5 \mathrm{mM} \mathrm{MgCl}_{2}, 200 \mu \mathrm{M}$ dNTPs (Promega), 10 pmol each of forward and reverse primers and $1 \mu 1$ of the genomic DNA. PCR was performed for 35 cycles with denaturation at $94^{\circ} \mathrm{C}$ for $30 \mathrm{sec}$; primer annealing at $61^{\circ} \mathrm{C}$ for $1 \mathrm{~min}$ and primer extension at $72^{\circ} \mathrm{C}$ for $1 \mathrm{~min} 30 \mathrm{sec}$. An initial denaturation of $94^{\circ} \mathrm{C}$ for 2 min was also included in the PCR reaction. The PCR products were analysed in $1.2 \%$ agarose gel.

DNA sequencing. Smad7 exon 4 PCR product was eluted out from the gel using MoBio Gel extraction kit and by following 
the manufacturer's instructions. Sequencing of all the samples (and resequencing of selected samples) was carried out in an ABI 3730 capillary sequencer.

\section{Results}

Mutational analysis of Smad7 intragenic region encoding the Smad7 MH2 domain. Genomic DNA was extracted from the 60 clinical samples of cervical carcinoma. Exon 4 of Smad7 amplified from this purified DNA by PCR, was subject to direct sequencing. Exon 4 was selected for analysis since it encodes the Mad Homology 2 (MH2) domain of Smad7, the domain that is believed to be critical for interaction with the cytoplasmic domain of type I TGF- 3 receptors (23). Also, previous site-directed mutagenesis studies of Smad7 indicate that mutations of certain key residues of the $\mathrm{MH} 2$ domain can impair Smad7 function (23). In order to study and compare the predominance of Smad7 genetic variants/mutations between high-risk HPV-positive and high-risk HPV-negative tumors, half of the clinical samples were selected to be oncogenic HPV-positive and the rest oncogenic HPV-negative. Histopathological diagnosis of these biopsies was invasive squamous cell carcinoma in all the cases.

We found a $\mathrm{G}$ to $\mathrm{C}$ change involving codon 391 in two of the 60 samples $(3.3 \%)$. Occurring at the third position of the codon, this $\mathrm{G}$ to $\mathrm{C}$ change does not cause any change at the amino acid level. Analysis of the chromatogram of this region clearly shows that the consensus sequence is also present. Since the signal intensities of the two forms are approximately equal, the variant likely exists in the heterozygous state in both the samples. However, this genetic variant (corresponding to position 1460 of Smad7 mRNA) has been identified previously as a synonymous single nucleotide polymorphism, found in the heterozygous state (dbSNP cluster id rs34151545).

No genetic aberration was observed in the set of analyzed tumor tissues. Results are presented in Table I. Chromatograms of the variants along with their consensus counterparts are shown in Fig. 1A and B.

\section{Discussion}

Mutations in genes for TGF- $\beta$, TGF- $\beta$ receptors and Smads have been reported in different cancers including cervical cancer (11-13). While Smad2, Smad3 and Smad4 have been screened in cervical carcinomas, mutational analysis of Smad7 has not been carried out in cervical cancer. It is of interest to note that mutational analysis of Smad7 has been carried out in colorectal cancer, hepatocellular carcinoma, ovarian cancer and in pancreatic cancer (25-29). Although polymorphic variants of Smad7 have been described in some of these studies, no abnormalities of Smad7 have yet been identified in these cancers. Another recent study reported the significant association of common Smad7 alleles with colorectal cancer risk (29). We screened for mutations in exon 4 of Smad7 in 30 oncogenic HPV-positive cervical cancers and in 30 oncogenic HPV-negative cervical carcinomas.

The heterozygous $\mathrm{G}$ to $\mathrm{C}$ variant in codon 391 found in this study is synonymous and has been identified previously. The variant allele, discovered in the Caucasian population, was reported in the database of single nucleotide polymorphisms
(dbSNP at NCBI), as part of the International Hap Map. Although the variant has been dubbed 'silent', the possibility of the change affecting protein structure and expression levels cannot be ruled out. Phenotype association studies involving this allele are warranted.

The fact that no genetic aberration was detected in any of the 60 cervical cancer samples, strongly suggest that mutations of Smad7 are unlikely to be of etiological significance as far as human cervical cancer is concerned.

\section{Acknowledgements}

Ramkumar Hariharan is supported by a research fellowship from the Council of Scientific and Industrial Research (CSIR), Government of India [F. No. 9/553 (14)/2003].

\section{References}

1. Nair S and Pillai MR: Human papillomavirus and disease mechanisms: relevance to oral and cervical cancers. Oral Dis 11: 350-359, 2005.

2. Schiffman M, Castle PE, Jeronimo J, Rodriguez AC and Wacholder S: Human papillomavirus and cervical cancer. Lancet 370: 890-907, 2007.

3. Bosch FX and de Sanjosé S: The epidemiology of human papillomavirus infection and cervical cancer. Dis Markers 23: 213-227, 2007.

4. Tsunokawa Y, Takebe N, Kasamatsu T, Terada M and Sugimura T: Transforming activity of human papillomavirus type 16 DNA sequence in a cervical cancer. Proc Natl Acad Sci USA 83: 2200-2203, 1986.

5. González Martín A: Molecular biology of cervical cancer. Clin Transl Oncol 9: 347-354, 2007.

6. Sakaguchi J, Kyo S, Kanaya T, et al: Aberrant expression and mutations of TGF-beta receptor type II gene in endometrial cancer. Gynecol Oncol 98: 427-433, 2005.

7. Musch A, Rabe C, Paik MD, et al: Altered expression of TGF-beta receptors in hepatocellular carcinoma - effects of a constitutively active TGF-beta type I receptor mutant. Digestion 71: 781-791, 2005.

8. Lazzereschi D, Nardi F, Turco A, et al: A complex pattern of mutations and abnormal splicing of Smad4 is present in thyroid tumours. Oncogene 24: 5344-5354, 2005.

9. Chen T, Jackson CR, Link A, et al: Int7G24A variant of transforming growth factor-beta receptor type I is associated with invasive breast cancer. Clin Cancer Res 12: 392-397, 2007.

10. Chen T, Jackson C, Costello B, et al: An intronic variant of the TGFBR1 gene is associated with carcinomas of the kidney and bladder. Int J Cancer 112: 420-425, 2004.

11. Baldus SE, Schwarz E, Lohrey C, et al: Smad4 deficiency in cervical carcinoma cells. Oncogene 24: 810-819, 2005.

12. Maliekal TT, Anto RJ and Karunagaran D: Differential activation of Smads in HeLa and $\mathrm{SiHa}$ cells that differ in their response to transforming growth factor-beta. J Biol Chem 279: 36287-36292, 2004.

13. Maliekal TT, Antony ML, Nair A, Paulmurugan R and Karunagaran D: Loss of expression, and mutations of Smad 2 and Smad 4 in human cervical cancer. Oncogene 22: 4889-4897, 2003.

14. Attisano L and Wrana JL: Smads as transcriptional co-modulators. Curr Opin Cell 24: 235-243, 2000.

15. Massague J, Blain SW and Lo RS: TGF beta signaling in growth control, cancer and heritable disorders. Cell 103: 295-309, 2000 .

16. Wrana JL: Regulation of Smad activity. Cell 100: 189-192, 2000.

17. Ten Dijke P, Miyazono K and Heldin CH: Signaling inputs converge on nuclear effectors in TGF-beta signaling. Trends Biochem Sci 25: 64-70, 2000.

18. Imamura T, Takase M, Nishihara A, et al: Smad6 inhibits signaling by the TGF-beta superfamily. Nature 389: 622-626, 1997.

19. Hayashi H, Abdollah S, Qiu Y, et al: The MAD-related protein Smad7 associates with the TGF-beta receptor and functions as an antagonist of TGF-beta signaling. Cell 89: 1165-1173, 1997. 
20. Ishisaki A, Yamato K, Hashimoto S, et al: Differential inhibition of Smad6 and Smad7 on bone morphogenetic protein - and activin-mediated growth arrest and apoptosis in B cells. J Biol Chem 274: 13637-13642, 1999.

21. Itoh S, Landström M, Hermansson A, et al: Transforming growth factor 1 induces nuclear export of inhibitory Smad7. J Biol Chem 273: 29195-29201, 1998.

22. Nakayama T, Berg LK and Christian JL: Dissection of inhibitory Smad proteins: both $\mathrm{N}$ - and $\mathrm{C}$-terminal domains are necessary for full activities of Xenopus Smad6 and Smad7. Mech Dev 100: 251-262, 2001

23. Mochizuki T, Miyazaki H, Hara T, et al: Roles for the MH2 domain of Smad7 in the specific inhibition of transforming growth factor-beta superfamily signaling. J Biol Chem 279: 31568-31574, 2004

24. Hu L, Guo M, He Z, Thornton J, McDaniel LS and Hughson MD: Human papillomavirus genotyping and p16INK4a expression in cervical intraepithelial neoplasia of adolescents. Mod Pathol 18: 267-273, 2005
25. Fukushima T, Mashiko M, Takita K, et al: Mutational analysis of TGF-beta type II receptor, Smad2, Smad3, Smad4, Smad6 and Smad7 genes in colorectal cancer. J Exp Clin Cancer Res 22: 315-320, 2003

26. Kawate S, Ohwada S, Hamada K, et al: Mutational analysis of the Smad6 and Smad7 genes in hepatocellular carcinoma. Int J Mol Med 8: 49-52, 2001.

27. Wang D, Kanuma T, Mizumuma H, Ibuki Y and Takenoshita S: Mutation analysis of the Smad6 and Smad7 gene in human ovarian cancers. Int J Oncol 17: 1087-1091, 2000

28. Jonson T, Gorunova L, Dawiskiba S, et al: Molecular analyses of the $15 q$ and $18 \mathrm{q}$ SMAD genes in pancreatic cancer. Genes Chromosomes Cancer 24: 62-71, 1999.

29. Broderick P, Carvajal-Carmona L, Pittman AM, et al: A genome-wide association study shows that common alleles of SMAD7 influence colorectal cancer risk. Nat Genet 39: 1315-1317, 2007. 